Article

\title{
Palladium-Catalyzed Direct Addition of 2-Aminobenzonitriles to Sodium Arylsulfinates: Synthesis of o-Aminobenzophenones
}

\author{
Jiuxi Chen ${ }^{1,2}$, Jianjun $\mathrm{Li}^{1}$ and Weike $\mathrm{Su}^{1, *}$
}

1 Collaborative Innovation Center of Yangtze River Delta Region Green Pharmaceuticals, College of Pharmaceutical Sciences, Zhejiang University of Technology, Hangzhou 310014, China; E-Mails: jiuxichen@163.com (C.J.); lijjun@126.com (J.L.)

2 College of Chemistry \& Materials Engineering, Wenzhou University, Wenzhou 325035, China

* Authors to whom correspondence should be addressed; E-Mail: pharmlab@zjut.edu.cn; Tel./Fax: +86-571-8882-0899.

Received: 3 May 2014; in revised form: 15 May 2014 / Accepted: 19 May 2014 /

Published: 20 May 2014

\begin{abstract}
The first example of the palladium-catalyzed synthesis of $o$-aminobenzophenones in moderate to excellent yields via a direct addition of sodium arylsulfinates to unprotected 2-aminobenzonitriles was reported. A plausible mechanism for the formation of $o$-aminobenzophenones involving desulfination and addition reactions was proposed. The utility of this transformation was demonstrated by its compatibility with a wide range of functional groups. Thus, the method represents a convenient and practical strategy for synthesis of $o$-aminobenzophenones.
\end{abstract}

Keywords: palladium; 2-aminobenzonitriles; arylsulfinates; $o$-aminobenzophenones

\section{Introduction}

$o$-Aminobenzophenones have drawn much attention due to their various pharmaceutical activities in medicine chemistry [1-3], their use as versatile intermediates for further transformations in synthetic chemistry [4-8] and their application in materials chemistry [9]. As a consequence, wide demands for diverse $o$-aminobenzophenones in various fields have promoted the development of practical and diversified synthetic methods [10-15]. Recently, Mateos reported the addition reaction of Grignard reagents to 2-aminobenzonitrile for the construction of $o$-aminobenzophenone using continuous flow chemistry [16]; however, the rigorous conditions have restricted its application and substrate diversity. 
Compared with Grignard reagents, sodium arylsulfinates are relatively stable, easy to handle, and are generally used as the aryl source in transition-metal-catalyzed desulfinative reactions [17-21]. On the other hand, transformations of nitriles play an important role in both the laboratory and industry due to their well-recognized chemical versatility [22,23]. However, the nitrile group is generally inert in organometallic reactions, and thus acetonitrile or benzonitrile usually participate as solvents or ligands [24] in metal-catalyzed reactions. The Larock group [25] pioneered the addition of arylpalladium species to the cyano group. Since then, transition metal-catalyzed addition reactions of arylation reagents to nitriles have been developed [26-30]. Recently, we reported the palladiumcatalyzed addition of organoboron reagents to aliphatic nitriles for the preparation of alkyl aryl ketones, diketone compounds, and 2-arylbenzo[b]furans [31,32]. However, there is a major limitation in that trace or low yields of the desired products were observed when the substrates bore a free amino group; therefore developing a new catalyst system that would allow for the efficient reaction of problematic substrate combinations is highly desirable. These reasons may be due to side reactions and catalyst deactivation in the presence of the free amino group. In addition, nitriles bearing an electrondonating amino group, are less electrophilic, and hence addition of arylpalladium species to the cyano group ocurrs more slowly than with their electron-neutral analogues. We envisioned that electrophiles might exhibit greatly enhanced reactivity due to the formation of stable, weak coordinating and electron withdrawing cationic species by adding an appropriate additive to the reaction system.

To the best of our knowledge, examples of $o$-aminobenzophenone synthesis using sodium arylsulfinates as coupling partners have never been reported. As part of the continuing efforts in our laboratory toward the development of palladium-catalyzed addition reactions [31-37], herein we report a simple and efficient protocol for the synthesis of $o$-aminobenzophenones by palladium-catalyzed direct addition of sodium arylsulfinates to unprotected 2-aminobenzonitriles (Scheme 1).

Scheme 1. Pd-catalyzed addition of arylsulfinates to 2-aminobenzonitriles.
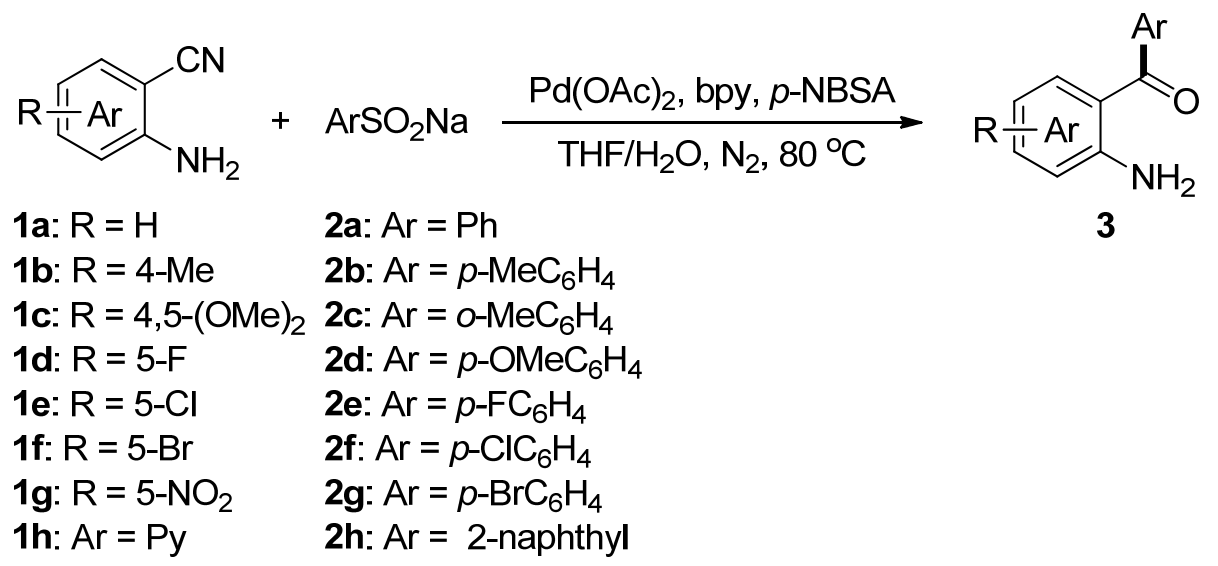

3

\section{Results and Discussion}

We began our study by examining the reaction between 2-aminobenzonitrile (1a) and sodium benzenesulfinate (2a) to establish the optimal reaction conditions (Table 1). On the basis of our previous addition protocol of organoborons to nitriles [31] a test reaction with $\mathrm{Pd}\left(\mathrm{O}_{2} \mathrm{CCF}_{3}\right)_{2}$ and 2,2'-bipyridine (bpy) as the catalytic system was performed under an air atmosphere. To our delight, 
the desired product $o$-aminobenzophenone (3a) was isolated in $18 \%$ yield (Table 1 , entry 1 ). Encouraged by this promising result, a series of trial experiments were performed in the presence of palladium catalysts and with adjustments to the reaction parameters in order to obtain more satisfactory results. First, we investigated different palladium catalysts. Among the palladium sources used, $\mathrm{Pd}(\mathrm{OAc})_{2}$ exhibited the highest catalytic reactivity, with $32 \%$ yield (Table 1, entries 1-6). Subsequently, various additives were examined in this transformation. Screening revealed that the use of $p$-nitrobenzene-sulfonic acid ( $p$-NBSA) as the additive that achieved the best result (73\% yield, Table 1, entry 11). Other additives, including $\mathrm{CF}_{3} \mathrm{CO}_{2} \mathrm{H}, \mathrm{CH}_{3} \mathrm{CO}_{2} \mathrm{H}, \mathrm{CH}_{3} \mathrm{SO}_{3} \mathrm{H}, \mathrm{PhSO}_{3} \mathrm{H}$ and $p$-toluenesulfonic acid ( $p$-TSA), were less efficient (Table 1, entries 1, 7-10). We next examined the solvent effect and found that THF or 2-MeTHF were superior to dioxane, toluene, and DMF (Table 1, entries 11-15). We were pleased to discover that only when the model reaction was performed in THF under a $\mathrm{N}_{2}$ atmosphere did the yield dramatically increase to $91 \%$ yield (Table 1, entry 16 ).

Table 1. Optimization of the reaction conditions ${ }^{\mathrm{a}}$.

\begin{tabular}{|c|c|c|c|c|}
\hline & $1 a$ & & 3 & \\
\hline Entry & Pd source & Additive & Solvent & Yield (\%) ${ }^{b}$ \\
\hline 1 & $\mathrm{Pd}\left(\mathrm{CF}_{3} \mathrm{CO}_{2}\right)_{2}$ & $\mathrm{CF}_{3} \mathrm{CO}_{2} \mathrm{H}$ & THF & 18 \\
\hline 2 & $\mathrm{PdCl}_{2}$ & $\mathrm{CF}_{3} \mathrm{CO}_{2} \mathrm{H}$ & THF & 11 \\
\hline 3 & $\mathrm{Pd}(\mathrm{OAc})_{2}$ & $\mathrm{CF}_{3} \mathrm{CO}_{2} \mathrm{H}$ & THF & 32 \\
\hline 4 & $\mathrm{Pd}(\mathrm{acac})_{2}$ & $\mathrm{CF}_{3} \mathrm{CO}_{2} \mathrm{H}$ & THF & 13 \\
\hline 5 & $\mathrm{Pd}\left(\mathrm{PPh}_{3}\right)_{4}$ & $\mathrm{CF}_{3} \mathrm{CO}_{2} \mathrm{H}$ & THF & trace \\
\hline 6 & $\mathrm{PdCl}_{2}$ (dppe) & $\mathrm{CF}_{3} \mathrm{CO}_{2} \mathrm{H}$ & THF & 0 \\
\hline 7 & $\mathrm{Pd}(\mathrm{OAc})_{2}$ & $\mathrm{CH}_{3} \mathrm{CO}_{2} \mathrm{H}$ & THF & 10 \\
\hline 8 & $\mathrm{Pd}(\mathrm{OAc})_{2}$ & $\mathrm{CH}_{3} \mathrm{SO}_{3} \mathrm{H}$ & THF & 44 \\
\hline 9 & $\mathrm{Pd}(\mathrm{OAc})_{2}$ & $\mathrm{PhSO}_{3} \mathrm{H}$ & THF & 59 \\
\hline 10 & $\mathrm{Pd}(\mathrm{OAc})_{2}$ & $p-\mathrm{TSA}^{\mathrm{c}}$ & THF & 61 \\
\hline 11 & $\mathrm{Pd}(\mathrm{OAc})_{2}$ & $p$-NBSA ${ }^{\mathrm{d}}$ & THF & 73 \\
\hline 12 & $\mathrm{Pd}(\mathrm{OAc})_{2}$ & $p$-NBSA & toluene & 42 \\
\hline 13 & $\mathrm{Pd}(\mathrm{OAc})_{2}$ & $p$-NBSA & 2-MeTHF & 69 \\
\hline 14 & $\mathrm{Pd}(\mathrm{OAc})_{2}$ & $p$-NBSA & dioxane & 53 \\
\hline 15 & $\mathrm{Pd}(\mathrm{OAc})_{2}$ & $p$-NBSA & DMF & trace \\
\hline 16 & $\mathrm{Pd}(\mathrm{OAc})_{2}$ & $p$-NBSA & THF & $91^{\mathrm{e}}$ \\
\hline
\end{tabular}

With the optimized reaction conditions in hand, we next explored the substrate scope of the addition reaction of 2-aminobenzonitriles $\mathbf{1}$ with sodium arylsulfinates $\mathbf{2}$ as shown in Scheme 1 . 
First, the addition reaction of 2-aminobenzonitrile (1a) with various sodium arylsulfinates $\mathbf{2 a}-\mathbf{h}$ was investigated under our standard conditions (Table 2). The mono-substituent positions of the phenyl moiety of sodium arylsulfinates were evaluated, and the results demonstrated that steric effects of substituents had an obvious impact on the yield of the reaction. For example, the addition reaction of 1a with para- and ortho-tolylsulfinate ( $2 \mathrm{~b}$ and $2 \mathrm{c}$ ) provided $87 \%$ of $\mathbf{3 b}$, while the yield of $\mathbf{3 c}$ decreased to $64 \%$ (Table 2, entries 2-3). The electronic properties of the substituents on the phenyl ring of the sodium arylsulfinates also affected the yields of the reaction to some extent. In general, the sodium arylsulfinates bearing an electron-donating substituent (e.g., - Me and - OMe) produced slightly higher yields than those analogues bearing an electron-withdrawing substituent (e.g., $-\mathrm{F},-\mathrm{Cl}$ and $-\mathrm{Br}$ ) (Table 2, entries 2, 4-7). Substrate $\mathbf{2 h}$, bearing a naphthyl group, was treated with $\mathbf{1 a}$ to deliver the desired product $\mathbf{3 h}$ in $90 \%$ yield (Table 2, entry 8 ). It is noteworthy that the fluoro, chloro, and bromo moieties (commonly used for cross-coupling reactions) in substrates were all tolerated and afforded several halogen-containing products 3e-g (Table 2, entries 5-7) in acceptable yields, leading to a useful handle for further cross-coupling reactions. However, treatment of an alkylsulfinate such as sodium methanesulfinate with 1a under the optimized conditions afforded only a trace amount of the desired product.

Table 2. Substrate scope of sodium arylsulfinates a

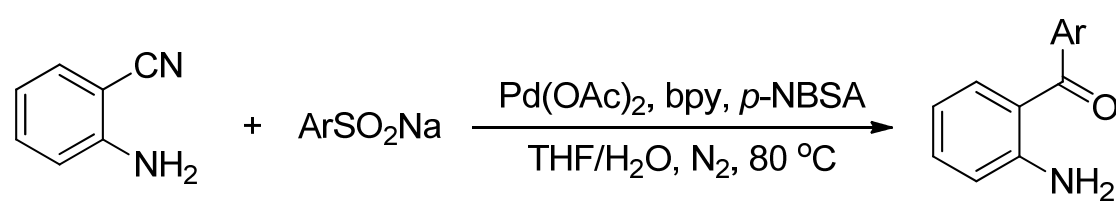

$1 \mathrm{a}$

2

3

\begin{tabular}{|c|c|c|c|}
\hline Entry & $\mathrm{ArSO}_{2} \mathrm{Na}(2)$ & Product (3) & Yield $(\%)^{b}$ \\
\hline 1 & ${ }_{2} \mathrm{Na} \quad(2 \mathrm{a})$ & $3 \mathbf{a}$ & 91 \\
\hline 2 & $\mathrm{O}_{2} \mathrm{Na} \quad(\mathbf{2 b})$ & $3 b$ & 88 \\
\hline 3 & (2c) & $3 c$ & 64 \\
\hline 4 & $\mathrm{O}_{2} \mathrm{Na} \quad(\mathbf{2 d})$ & 3d & 85 \\
\hline 5 & $\mathrm{O}_{2} \mathrm{Na} \quad(\mathbf{2 e})$ & $3 e$ & 81 \\
\hline 6 & $\mathrm{SO}_{2} \mathrm{Na} \quad(2 \mathrm{f})$ & $3 f$ & 83 \\
\hline 7 & $\mathrm{SO}_{2} \mathrm{Na} \quad(2 \mathrm{~g})$ & $3 g$ & 80 \\
\hline 8 & (2h) & $3 \mathbf{h}$ & 90 \\
\hline
\end{tabular}

${ }^{\mathrm{a}}$ Reaction conditions: 1a $(0.3 \mathrm{mmol}), 2(0.6 \mathrm{mmol}), \mathrm{Pd}(\mathrm{OAc})_{2}(10 \mathrm{~mol} \%)$, bpy $(20 \mathrm{~mol} \%), p$-NBSA (3 mmol), THF $(2 \mathrm{~mL}), \mathrm{H}_{2} \mathrm{O}(1 \mathrm{~mL}), 80^{\circ} \mathrm{C}, 48 \mathrm{~h}, \mathrm{~N}_{2}$; ${ }^{\mathrm{b}}$ Isolated yield. 
Next, we turned our attention to the effect of the reactions of sodium benzenesulfinate (2a) with various 2-aminobenzonitriles $(\mathbf{1} \mathbf{a}-\mathbf{h})$ under our standard conditions and the results are summarized in Scheme 2. As expected, the groups on the phenyl ring of 2-aminobenzonitriles, such as methyl, methoxy, fluoro, chloro, bromo, and nitro were quite compatible. The electronic properties of the groups on the phenyl ring moiety of 2-aminobenzonitriles had little effect on the reaction. For example, substrates $\mathbf{1 b}$ and 1c bearing an electron-donating substituent (e.g., $-\mathrm{Me}$ or $-\mathrm{OMe}$ ), reacted with 2a smoothly and afforded the corresponding products $\mathbf{3 i}$ and $\mathbf{3} \mathbf{j}$ in $93 \%$ and $90 \%$ yields, respectively (Scheme 2, entries 2-3). Substrates 1d, 1e, 1f and $\mathbf{1 g}$ bearing an electron-withdrawing substituent (e.g., $-\mathrm{F},-\mathrm{Cl},-\mathrm{Br}$ and $-\mathrm{NO}_{2}$ ) were treated with $\mathbf{2 a}$ to afford $89 \%, 92 \%, 90 \%$ and 96\% yields of 3k, 31, 3m and 3n, respectively (Scheme 2, entries 4-7). Gratifyingly, the substrate 2-aminonicotinonitrile $(\mathbf{1 h})$, bearing a heteroaryl group underwent the reaction smoothly to afford the corresponding product 30 in $83 \%$ yield (Scheme 2, entry 8 ).

Scheme 2. Substrate scope of 2-aminobenzonitriles ${ }^{\text {a }}$.

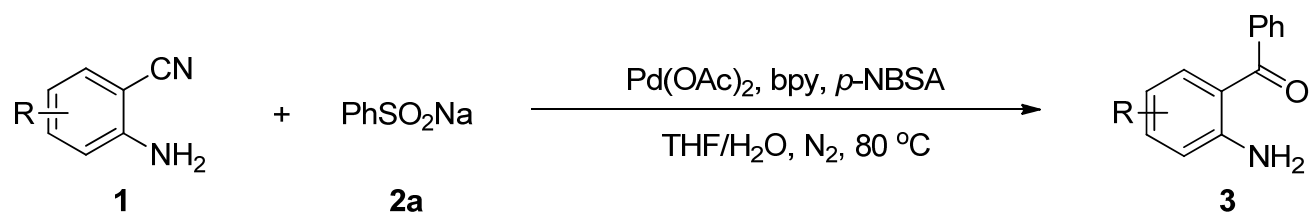

(1)<smiles>Nc1ccccc1C(=O)c1ccccc1</smiles>

$3 \mathbf{a}(91 \%)$

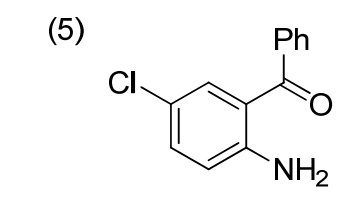

$3 \mathbf{l}(92 \%)$
(2)<smiles>Cc1ccc(C(=O)c2ccccc2)c(N)c1</smiles>

$3 \mathbf{i}(93 \%)$
(3)<smiles>COc1cc(N)c(C(=O)c2ccccc2)cc1OC</smiles>

3j (90\%)
(4)<smiles>Nc1ccc(F)cc1C(=O)c1ccccc1</smiles>

3k $(89 \%)$
(6)

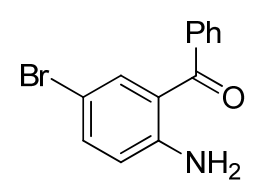

$3 \mathrm{~m}(90 \%)$
(7)<smiles>Nc1ccc([N+](=O)[O-])cc1C(=O)c1ccccc1</smiles>

3n (96\%)
(8)<smiles>Nc1ncccc1C(=O)c1ccccc1</smiles>

30 (83\%)

${ }^{a}$ Reaction conditions: 1 (0.3 mmol), 2a (0.6 mmol), Pd(OAc) $)_{2}(10 \mathrm{~mol} \%)$, bpy (20 mol\%), p-NBSA ( $3 \mathrm{mmol})$, THF $(2 \mathrm{~mL}), \mathrm{H}_{2} \mathrm{O}(1 \mathrm{~mL}), 80^{\circ} \mathrm{C}, 48 \mathrm{~h}, \mathrm{~N}_{2}$; Isolated yield was given in parenthesis.

A plausible mechanism for the formation of $o$-aminobenzophenones is proposed in Scheme 3 . The following key steps are included in the catalytic pathway: (i) coordination of $\mathrm{Pd}(\mathrm{OAc})_{2}$ with arylsulfinic acids (or sodium arylsulfinates) to afford a palladium species $\mathbf{A}$; (ii) the desulfination of the arylsulfinic acid to give aryl-palladium species $\mathbf{B}$; (iii) the formation of intermediate $\mathbf{D}$ by the coordination of species B with cyano group in 2-cyanobenzenaminium (C); (iv) carbopalladation of the 2-aminobenzonitriles to produce the corresponding ketimine complex $\mathbf{E}$; (v) protonation of the ketimine complex $\mathbf{E}$ to afford the ketimine intermediate $\mathbf{F}$ and regenerate an active palladium species. Hydrolysis of the ketimine intermediate $\mathbf{F}$ delivers the corresponding $o$-aminobenzophenones as the desired products. 
Scheme 3. Proposed mechanism.

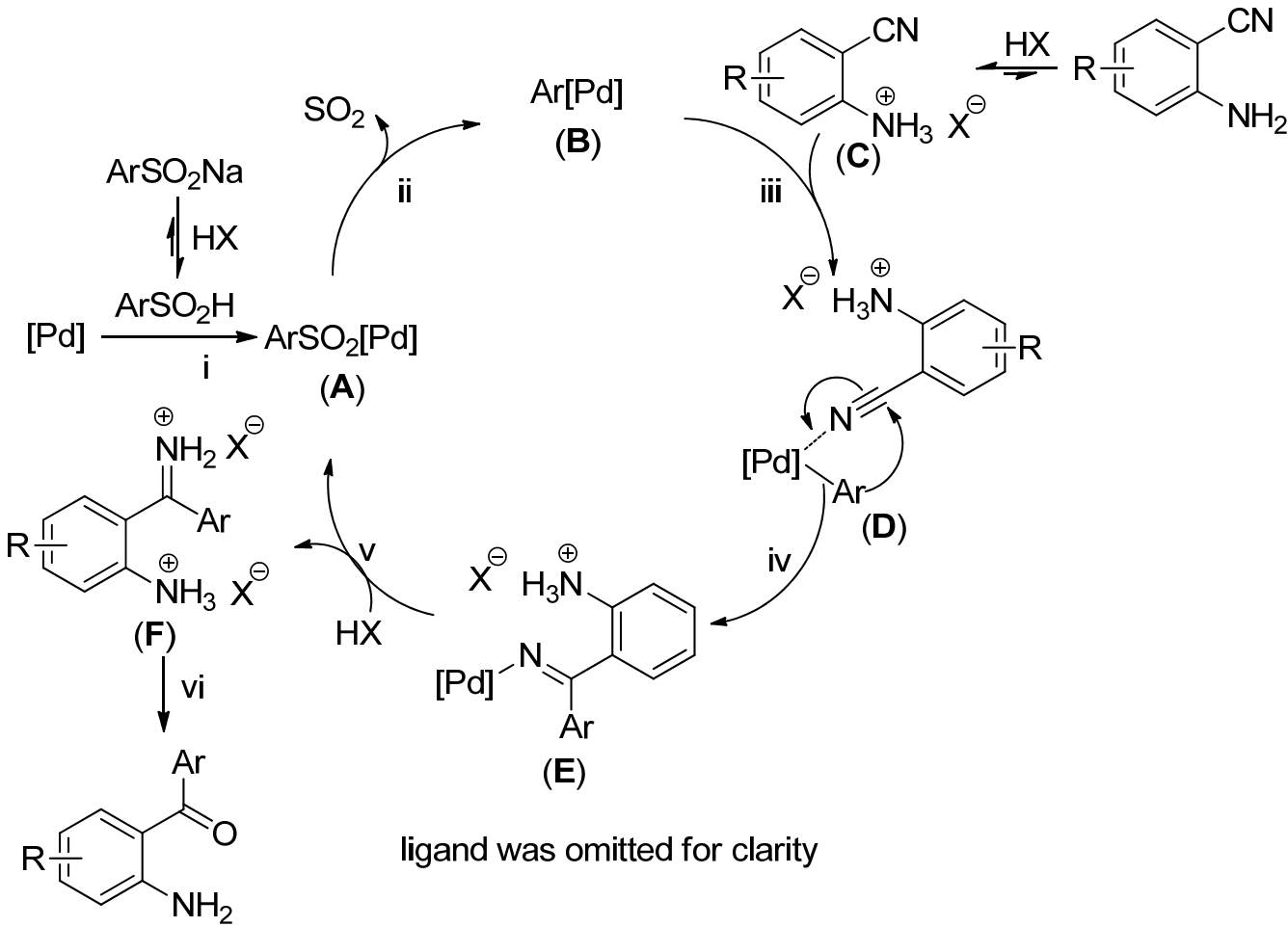

\section{Experimental}

\section{General Information}

Melting points are uncorrected. ${ }^{1} \mathrm{H}-\mathrm{NMR}$ and ${ }^{13} \mathrm{C}-\mathrm{NMR}$ spectra were measured on a $500 \mathrm{MHz}$ spectrometer using $\mathrm{CDCl}_{3}$ as the solvent with tetramethylsilane (TMS) as an internal standard at room temperature. Chemical shifts are given $\mathrm{n} \delta$ relative to TMS, and the coupling constants $J$ are given in hertz. Other commercially obtained reagents were used without further purification. All reactions under $\mathrm{N}_{2}$ atmosphere were conducted using standard Schlenk techniques. Column chromatography was performed using EM silica gel 60 (300-400 mesh).

\section{General Procedure for the Synthesis of o-Aminobenzophenones}

Under a $\mathrm{N}_{2}$ atmosphere, a Schlenk tube was charged with 2-aminobenzonitrile 1 (0.3 mmol), sodium arylsulfinate $2(0.6 \mathrm{mmol}), \mathrm{Pd}(\mathrm{OAc})_{2}(10 \mathrm{~mol} \%)$, bpy $(20 \mathrm{~mol} \%), p$-NBSA (10 equiv), THF $(2 \mathrm{~mL})$, and $\mathrm{H}_{2} \mathrm{O}(1 \mathrm{~mL})$ at room temperature. The reaction mixture was stirred vigorously at $80{ }^{\circ} \mathrm{C}$ for $48 \mathrm{~h}$. The mixture was poured into ethyl acetate, which was washed with saturated $\mathrm{NaHCO}_{3}(2 \times 10 \mathrm{~mL})$ and then brine $(1 \times 10 \mathrm{~mL})$. After the aqueous layer was extracted with ethyl acetate, the combined organic layers were dried over anhydrous $\mathrm{MgSO}_{4}$ and evaporated under reduced pressure. The residue was purified by flash column chromatography (hexane/ethyl acetate) to afford the desired products 3 .

2-Aminobenzophenone (3a). Pale yellow solid (91\% yield), mp $110-112{ }^{\circ} \mathrm{C}$ (Lit. [13] $109-111{ }^{\circ} \mathrm{C}$ ); ${ }^{1} \mathrm{H}-\mathrm{NMR}\left(\mathrm{CDCl}_{3}, 500 \mathrm{MHz}\right): \delta$ 7.63-7.64 (m, 2H), 7.31-7.54 (m, 4H), 7.26-7.28 (m, 1H), $6.74(\mathrm{~d}$, 
$J=8.3 \mathrm{~Hz}, 1 \mathrm{H}), 6.60(\mathrm{t}, J=7.6 \mathrm{~Hz}, 1 \mathrm{H}), 6.09(\mathrm{~s}, 2 \mathrm{H}) ;{ }^{13} \mathrm{C}-\mathrm{NMR}\left(\mathrm{CDCl}_{3}, 125 \mathrm{MHz}\right) \delta 199.1,150.9$, 140.1, 134.6, 134.2, 131.0, 129.1, 128.1, 118.2, 117.0, 115.5 .

(2-Aminophenyl)(p-tolyl)methanone (3b) [5]. Pale yellow solid ( $88 \%$ yield), mp $92-93{ }^{\circ} \mathrm{C}$ (not reported); ${ }^{1} \mathrm{H}-\mathrm{NMR}\left(\mathrm{CDCl}_{3}, 500 \mathrm{MHz}\right): \delta 7.56(\mathrm{~d}, J=8.1 \mathrm{~Hz}, 2 \mathrm{H}), 7.45(\mathrm{~d}, J=8.0 \mathrm{~Hz}, 1 \mathrm{H}), 7.25-7.30(\mathrm{~m}, 3 \mathrm{H})$, $6.73(\mathrm{~d}, J=8.2 \mathrm{~Hz}, 1 \mathrm{H}), 6.60(\mathrm{t}, J=7.5 \mathrm{~Hz}, 1 \mathrm{H}), 6.00(\mathrm{~s}, 2 \mathrm{H}), 2.42(\mathrm{~s}, 3 \mathrm{H}) ;{ }^{13} \mathrm{C}-\mathrm{NMR}\left(\mathrm{CDCl}_{3}\right.$, $125 \mathrm{MHz}) \delta 198.8,150.7,141.7,137.2,134.4,134.0,129.4,128.7,118.6,116.9,115.5,21.5$.

(2-Aminophenyl)(o-tolyl)methanone (3c). Pale yellow solid (64\% yield), mp $79-81{ }^{\circ} \mathrm{C}$ (Lit. [38] $\left.84{ }^{\circ} \mathrm{C}\right) ;{ }^{1} \mathrm{H}-\mathrm{NMR}\left(\mathrm{CDCl}_{3}, 500 \mathrm{MHz}\right): \delta 7.32-7.35(\mathrm{~m}, 1 \mathrm{H}), 7.20-7.29(\mathrm{~m}, 5 \mathrm{H}), 6.71(\mathrm{~d}, J=8.3 \mathrm{~Hz}$, $1 \mathrm{H}), 6.52(\mathrm{t}, J=7.6 \mathrm{~Hz}, 1 \mathrm{H}), 6.41(\mathrm{~s}, 2 \mathrm{H}), 2.27(\mathrm{~s}, 3 \mathrm{H}) ;{ }^{13} \mathrm{C}-\mathrm{NMR}\left(\mathrm{CDCl}_{3}, 125 \mathrm{MHz}\right) \delta 200.8,150.7$, $140.1,134.6,134.3,134.2,130.0,128.7,126.6,124.7,117.9,116.4,115.1,19.0$.

(2-Aminophenyl)(4-methoxyphenyl)methanone (3d). Pale yellow solid (85\% yield), mp 77-78 ${ }^{\circ} \mathrm{C}$ (Lit. [4] 75-76 $\left.{ }^{\circ} \mathrm{C}\right) ;{ }^{1} \mathrm{H}-\mathrm{NMR}\left(\mathrm{CDCl}_{3}, 500 \mathrm{MHz}\right): \delta 7.68(\mathrm{~d}, J=8.8 \mathrm{~Hz}, 2 \mathrm{H}), 7.46(\mathrm{~d}, J=8.0 \mathrm{~Hz}, 1 \mathrm{H})$, $7.28(\mathrm{t}, J=7.7 \mathrm{~Hz}, 1 \mathrm{H}), 6.95(\mathrm{~d}, J=8.8 \mathrm{~Hz}, 2 \mathrm{H}), 6.73(\mathrm{~d}, J=8.3 \mathrm{~Hz}, 1 \mathrm{H}), 6.62(\mathrm{t}, J=7.1 \mathrm{~Hz}, 1 \mathrm{H})$, $5.86(\mathrm{~s}, 2 \mathrm{H}), 3.38(\mathrm{~s}, 3 \mathrm{H}) ;{ }^{13} \mathrm{C}-\mathrm{NMR}\left(\mathrm{CDCl}_{3}, 125 \mathrm{MHz}\right) \delta 197.8,162.3,150.4,134.0,133.7,131.8$, 122.2, 199.0, 117.0, 115.6, 113.4, 55.4.

(2-Aminophenyl)(4-fluorophenyl)methanone (3e) [6]. Pale yellow solid (81\% yield), mp $128-129{ }^{\circ} \mathrm{C}$ (not reported); ${ }^{1} \mathrm{H}-\mathrm{NMR}\left(\mathrm{CDCl}_{3}, 500 \mathrm{MHz}\right): \delta$ 7.67-7.69 (m, 2H), $7.42(\mathrm{~d}, J=8.1 \mathrm{~Hz}, 1 \mathrm{H}), 7.29-7.32$ $(\mathrm{m}, 1 \mathrm{H}), 7.14(\mathrm{t}, J=8.7 \mathrm{~Hz}, 2 \mathrm{H}), 6.75(\mathrm{~d}, J=8.3 \mathrm{~Hz}, 1 \mathrm{H}), 6.62(\mathrm{t}, J=7.6 \mathrm{~Hz}, 1 \mathrm{H}), 6.03(\mathrm{~s}, 2 \mathrm{H})$; ${ }^{13} \mathrm{C}-\mathrm{NMR}\left(\mathrm{CDCl}_{3}, 125 \mathrm{MHz}\right) \delta 197.5,165.5,163.5,150.8,136.1,134.3,134.2,131.7,131.6,118.1$, 117.1, 115.6, 115.3, 115.1.

(2-Aminophenyl)(4-chlorophenyl)methanone (3f) [5]. Pale yellow solid (83\% yield), mp 100-101 ${ }^{\circ} \mathrm{C}$ (not reported); ${ }^{1} \mathrm{H}-\mathrm{NMR}\left(\mathrm{CDCl}_{3}, 500 \mathrm{MHz}\right): \delta 7.59$ (d, $\left.J=8.5 \mathrm{~Hz}, 2 \mathrm{H}\right), 7.31-7.44(\mathrm{~m}, 3 \mathrm{H}), 7.26-7.28$ $(\mathrm{m}, 1 \mathrm{H}), 6.73(\mathrm{~d}, J=8.9 \mathrm{~Hz}, 1 \mathrm{H}), 6.60(\mathrm{t}, J=7.6 \mathrm{~Hz}, 1 \mathrm{H}), 6.08(\mathrm{~s}, 2 \mathrm{H}) ;{ }^{13} \mathrm{C}-\mathrm{NMR}\left(\mathrm{CDCl}_{3}, 125 \mathrm{MHz}\right)$ $\delta 197.9,151.0,138.4,137.4,134.5,134.3,130.6,128.4,117.9,117.2,115.7$.

(2-Aminophenyl)(4-bromophenyl)methanone (3g) [5]. Pale yellow solid (80\% yield), mp $109-111{ }^{\circ} \mathrm{C}$ (not reported); ${ }^{1} \mathrm{H}-\mathrm{NMR}\left(\mathrm{CDCl}_{3}, 500 \mathrm{MHz}\right): \delta 7.60(\mathrm{~d}, J=8.5 \mathrm{~Hz}, 2 \mathrm{H}), 7.51(\mathrm{~d}, J=8.5 \mathrm{~Hz}, 2 \mathrm{H}), 7.40$ $(\mathrm{d}, J=8.1 \mathrm{~Hz}, 1 \mathrm{H}), 7.27-7.32(\mathrm{~m}, 1 \mathrm{H}), 6.74(\mathrm{~d}, J=8.3 \mathrm{~Hz}, 1 \mathrm{H}), 6.61(\mathrm{t}, J=7.6 \mathrm{~Hz}, 1 \mathrm{H}), 6.10(\mathrm{~s}, 2 \mathrm{H})$; ${ }^{13} \mathrm{C}-\mathrm{NMR}\left(\mathrm{CDCl}_{3}, 125 \mathrm{MHz}\right) \delta 197.8,151.0,138.8,134.5,134.2,131.4,130.7,125.8,117.7,117.1$, 115.6.

(2-Aminophenyl)(naphthalen-2-yl)methanone (3h). Pale yellow solid (90\% yield), mp $107-108{ }^{\circ} \mathrm{C}$ (Lit. [38] $\left.106{ }^{\circ} \mathrm{C}\right) ;{ }^{1} \mathrm{H}-\mathrm{NMR}\left(\mathrm{CDCl}_{3}, 500 \mathrm{MHz}\right): \delta 8.12(\mathrm{~s}, 1 \mathrm{H}), 7.89-7.93(\mathrm{~m}, 3 \mathrm{H}), 7.77(\mathrm{~d}, J=8.5 \mathrm{~Hz}$, 1H), 7.51-7.60 (m, 3H), 7.30-7.31 (m, 1H), $6.77(\mathrm{~d}, J=8.3 \mathrm{~Hz}, 1 \mathrm{H}), 6.62(\mathrm{t}, J=7.1 \mathrm{~Hz}, 1 \mathrm{H}), 6.09$ (s, $2 \mathrm{H}) ;{ }^{13} \mathrm{C}-\mathrm{NMR}\left(\mathrm{CDCl}_{3}, 125 \mathrm{MHz}\right) \delta 199.0,150.9,137.3,134.6,134.6,134.2,132.3,130.1,129.1$, $128.0,127.8,127.7,126.7,125.8,118.5,117.1,115.6$.

(2-Amino-4-methylphenyl)(phenyl)methanone (3i) [5]. Pale yellow solid (93\% yield), mp 67-68 ${ }^{\circ} \mathrm{C}$ (not reported); ${ }^{1} \mathrm{H}-\mathrm{NMR}\left(\mathrm{CDCl}_{3}, 500 \mathrm{MHz}\right): \delta 7.61(\mathrm{~d}, J=8.4 \mathrm{~Hz}, 2 \mathrm{H}), 7.44-7.52(\mathrm{~m}, 3 \mathrm{H}), 7.33(\mathrm{~d}$, 
$J=8.2 \mathrm{~Hz}, 1 \mathrm{H}), 6.54(\mathrm{~s}, 1 \mathrm{H}), 6.41(\mathrm{~d}, J=7.3 \mathrm{~Hz}, 1 \mathrm{H}), 6.12(\mathrm{~s}, 2 \mathrm{H}), 2.29(\mathrm{~s}, 3 \mathrm{H}) ;{ }^{13} \mathrm{C}-\mathrm{NMR}\left(\mathrm{CDCl}_{3}\right.$, $125 \mathrm{MHz}) \delta 198.7,151.3,145.4,140.5,134.8,130.8,129.0,128.1,117.1,117.0,116.0,21.8$.

(2-Amino-4,5-dimethoxyphenyl)(phenyl)methanone (3j) [7]. Pale yellow solid (90\% yield), mp 79-81 ${ }^{\circ} \mathrm{C}$ (not reported); ${ }^{1} \mathrm{H}-\mathrm{NMR}\left(\mathrm{CDCl}_{3}, 500 \mathrm{MHz}\right): \delta 7.61(\mathrm{~d}, J=8.4 \mathrm{~Hz}, 2 \mathrm{H}), 7.43-7.51(\mathrm{~m}, 3 \mathrm{H}), 6.92$ $(\mathrm{s}, 1 \mathrm{H}), 6.25(\mathrm{~s}, 2 \mathrm{H}), 6.20(\mathrm{~s}, 1 \mathrm{H}), 3.88(\mathrm{~s}, 3 \mathrm{H}), 3.65(\mathrm{~s}, 3 \mathrm{H}) ;{ }^{13} \mathrm{C}-\mathrm{NMR}\left(\mathrm{CDCl}_{3}, 125 \mathrm{MHz}\right) \delta 197.2$, 155.5, 148.6, 140.7, 139.7, 130.6, 128.7, 128.1, 116.7, 110.0, 99.3, 56.6, 55.9.

(2-Amino-5-fluorophenyl)(phenyl)methanone (3k) [8]. Pale yellow solid (89\% yield), mp $117-118{ }^{\circ} \mathrm{C}$ (not reported); ${ }^{1} \mathrm{H}-\mathrm{NMR}\left(\mathrm{CDCl}_{3}, 500 \mathrm{MHz}\right): \delta$ 7.46-7.65 (m, 5H), 7.05-7.16 (m, 2H), 6.70-6.72 $(\mathrm{m}, 1 \mathrm{H}), 5.91(\mathrm{~s}, 2 \mathrm{H}) ;{ }^{13} \mathrm{C}-\mathrm{NMR}\left(\mathrm{CDCl}_{3}, 125 \mathrm{MHz}\right) \delta 198.0,154.1,152.2,147.3,139.4,131.5,129.1$, $128.3,122.3,122.1,119.1,118.9,118.2,118.1,117.9$.

(2-Amino-5-chlorophenyl)(phenyl)methanone (31) [5]. Pale yellow solid (92\% yield), mp 97-98 ${ }^{\circ} \mathrm{C}$ (not reported); ${ }^{1} \mathrm{H}-\mathrm{NMR}\left(\mathrm{CDCl}_{3}, 500 \mathrm{MHz}\right): \delta 7.63(\mathrm{~d}, J=8.4 \mathrm{~Hz}, 2 \mathrm{H}), 7.47-7.56(\mathrm{~m}, 3 \mathrm{H}), 7.41(\mathrm{~d}$, $J=2.5 \mathrm{~Hz}, 1 \mathrm{H}), 7.23-7.25(\mathrm{~m}, 1 \mathrm{H}), 6.69(\mathrm{~d}, J=8.8 \mathrm{~Hz}, 1 \mathrm{H}), 6.07(\mathrm{~s}, 2 \mathrm{H}) ;{ }^{13} \mathrm{C}-\mathrm{NMR}\left(\mathrm{CDCl}_{3}, 125 \mathrm{MHz}\right)$ $\delta 198.0,149.4,139.3,134.2,133.3,131.6,129.1,128.4,120.0,118.8,118.5$.

(2-Amino-5-bromophenyl)(phenyl)methanone (3m) [7]. Pale yellow solid (90\% yield), mp 109-110 ${ }^{\circ} \mathrm{C}$ (not reported); ${ }^{1} \mathrm{H}-\mathrm{NMR}\left(\mathrm{CDCl}_{3}, 500 \mathrm{MHz}\right): \delta 7.63(\mathrm{~d}, J=8.4 \mathrm{~Hz}, 2 \mathrm{H}), 7.47-7.58(\mathrm{~m}, 4 \mathrm{H}), 7.35-7.37$ $(\mathrm{m}, 1 \mathrm{H}), 6.65(\mathrm{~d}, J=8.8 \mathrm{~Hz}, 1 \mathrm{H}), 6.10(\mathrm{~s}, 2 \mathrm{H}) ;{ }^{13} \mathrm{C}-\mathrm{NMR}\left(\mathrm{CDCl}_{3}, 125 \mathrm{MHz}\right) \delta 198.0,149.8,139.4$, $136.9,136.3,131.7,129.2,128.5,119.6,118.9,106.7$.

(2-Amino-5-nitrophenyl)(phenyl)methanone (3n) [5]. Pale yellow solid (96\% yield), mp $151-152{ }^{\circ} \mathrm{C}$ (not reported); ${ }^{1} \mathrm{H}-\mathrm{NMR}\left(\mathrm{CDCl}_{3}, 500 \mathrm{MHz}\right): \delta 8.48(\mathrm{~s}, 1 \mathrm{H}), 8.17(\mathrm{~d}, J=9.2 \mathrm{~Hz}, 1 \mathrm{H}), 7.51-7.66(\mathrm{~m}, 5 \mathrm{H})$, $6.90(\mathrm{~s}, 2 \mathrm{H}), 6.76(\mathrm{~d}, J=9.2 \mathrm{~Hz}, 1 \mathrm{H}) ;{ }^{13} \mathrm{C}-\mathrm{NMR}\left(\mathrm{CDCl}_{3}, 125 \mathrm{MHz}\right) \delta 198.0,155.3,138.5,136.7$, $132.2,131.6,129.3,129.2,128.7,116.8,116.1$.

(2-Aminopyridin-3-yl)(phenyl)methanone (3o). Pale yellow solid (83\% yield), mp 143-144 ${ }^{\circ} \mathrm{C}$ (Lit. [39] $\left.140{ }^{\circ} \mathrm{C}\right) ;{ }^{1} \mathrm{H}-\mathrm{NMR}\left(\mathrm{CDCl}_{3}, 500 \mathrm{MHz}\right): \delta 8.25(\mathrm{~d}, J=4.8 \mathrm{~Hz}, 2 \mathrm{H}), 7.76-7.77(\mathrm{~m}, 1 \mathrm{H}), 7.48-7.62(\mathrm{~m}$, $5 \mathrm{H}), 6.82(\mathrm{~s}, 2 \mathrm{H}), 6.59-6.62(\mathrm{~m}, 1 \mathrm{H}) ;{ }^{13} \mathrm{C}-\mathrm{NMR}\left(\mathrm{CDCl}_{3}, 125 \mathrm{MHz}\right) \delta 197.8,159.8,153.9,143.0$, $139.2,131.6,129.1,128.4,112.9,112.1$.

\section{Conclusions}

In summary, we have developed a new strategy for constructing $o$-aminobenzophenones in moderate to excellent yields via palladium-catalyzed direct addition reaction of sodium arylsulfinates to unprotected 2-aminobenzonitriles. Further efforts to extend this catalytic system to the preparation of other useful compounds are currently underway in our laboratories.

\section{Supplementary Materials}

Supplementary materials can be accessed at: http://www.mdpi.com/1420-3049/19/5/6439/s1. 


\section{Acknowledgments}

We thank the National Natural Science Foundation of China (No. 21102105) and Zhejiang Provincial Natural Science Foundation (Nos. LY13B020015 and LY14B020009) for financial support.

\section{Author Contributions}

The contributions of the respective authors are as follows: Jiuxi Chen and Weike Su designed the experiments. Jiuxi Chen and Jianjun Li performed the experiments and developed the reactions. Jiuxi Chen had the idea for this work and prepared this manuscript with feedback from Jianjun Li and Weike Su.

\section{Conflicts of Interest}

The authors declare no conflict of interest.

\section{References}

1. Singh, R.K.; Prasad, D.N.; Bhardwaj, T.R. Design, synthesis and evaluation of aminobenzophenone derivatives containing nitrogen mustard moiety as potential central nervous system antitumor agent. Med. Chem. Res. 2013, 22, 5901-5911.

2. Liou, J.-P.; Chang, C.-W.; Song, J.-S.; Yang, Y.-N.; Yeh, C.-F.; Tseng, H.-Y.; Lo, Y.-K.; Chang, Y.-L.; Chang, C.-M.; Hsieh, H.-P. Synthesis and structure activity relationship of 2-aminobenzophenone derivatives as antimitotic agents. J. Med. Chem. 2002, 45, 2556-2562.

3. Castellano, S.; Taliani, S.; Viviano, M.; Milite, C.; da Pozzo, E.; Costa, B.; Barresi, E.; Bruno, A.; Cosconati, S.; Marinelli, L.; et al. Structure-activity relationship refinement and further assessment of 4-phenylquinazoline-2-carboxamide translocator protein ligands as antiproliferative agents in human glioblastoma tumors. J. Med. Chem. 2014, 57, 2413-2428.

4. Kobayashi, K.; Fujita, S.; Fukamachi, S.; Konishi, H. One-pot synthesis of quinoline-2(1H)-thiones from 2-isocyanostyrenes via electrocyclic reaction of the corresponding 2-isothiocyanatestyrenes. Synthesis 2009, 2009, 3378-3382.

5. Cai, S.; Zeng, J.; Bai, Y.; Liu, X. Access to Quinolines through gold-catalyzed intermolecular cycloaddition of 2-aminoaryl carbonyls and internal alkynes. J. Org. Chem. 2012, 77, 801-807.

6. Yan, Y.; Wang, Z. Metal-free intramolecular oxidative decarboxylative amination of primary a-amino acids with product selectivity. Chem. Commun. 2011, 47, 9513-9515.

7. Anand, N.; Reddy, K.H.P.; Satyanarayana, T.; Rao, K.S.R.; Burri, D.R. A magnetically recoverable $\gamma-\mathrm{Fe}_{2} \mathrm{O}_{3}$ nanocatalyst for the synthesis of 2-phenylquinazolines under solvent-free conditions. Catal. Sci. Technol. 2012, 2, 570-574.

8. Ren, L.; Lei, T.; Ye, J.; Gong, L. Step-economical synthesis of tetrahydroquinolines by asymmetric relay catalytic Friedläender condensation/transfer hydrogenation. Angew. Chem. Int. Ed. Engl. 2012, 51, 771-774.

9. Earmme, T.; Ahmed, E.; Jenekhe, S.A. Solution-processed highly efficient blue phosphorescent polymer light-emitting diodes enabled by a new electron transport material. Adv. Mater. 2010, 22 , 4744-4748. 
10. Walsh, D.A. The Synthesis of 2-aminobenzophenones. Synthesis 1980, 1980, 677-688.

11. Xie, Y.; Yang, Y.; Huang, L.; Zhang, X.; Zhang, Y. Pd-Catalyzed arylation/oxidation of benzylic C-H bond. Org. Lett. 2012, 14, 1238-1241.

12. Khedkar, M.V.; Tambade, P.J.; Qureshi, Z.S.; Bhanage, B.M. Pd/C: An efficient, heterogeneous and reusable catalyst for phosphane-free carbonylative suzuki coupling reactions of aryl and heteroaryl iodides. Eur. J. Org. Chem. 2010, 2010, 6981-6986.

13. Cai, M.; Peng, J.; Hao, W.; Ding, G. A phosphine-free carbonylative cross-coupling reaction of aryl iodides with arylboronic acids catalyzed by immobilization of palladium in MCM-41. Green Chem. 2011, 13, 190-196.

14. Mizuno, M.; Yamano, M. A new practical one-pot conversion of phenols to anilines. Org. Lett. 2005, 7, 3629-3631.

15. Thakur, K.G.; Ganapathy, D.; Sekar, G. D-Glucosamine as a green ligand for copper catalyzed synthesis of primary aryl amines from aryl halides and ammonia. Chem. Commun. 2011, 47, 5076-5078.

16. Mateos, C.; Rincón, J.A.; Villanueva, J. Efficient and scalable synthesis of ketones via nucleophilic Grignard addition to nitriles using continuous flow chemistry. Tetrahedron Lett. 2013, 45, 2226-2230.

17. Zhou, X.; Luo, J.; Liu, J.; Peng, S.; Deng, D. Pd-catalyzed desulfitative heck coupling with dioxygen as the terminal oxidant. Org. Lett. 2011, 13, 1432-1436.

18. Rao, H.; Yang, L.; Shuai, Q.; Li, C. Rhodium-catalyzed aerobic coupling between aldehydes and arenesulfinic acid salts: A novel synthesis of aryl ketones. Adv. Synth. Catal. 2011, 353, 1701-1706.

19. Chen, J.; Sun, Y.; Liu, B.; Liu, D.; Cheng, J. The palladium-catalyzed desulfitative cyanation of arenesulfonyl chlorides and sodium sulfinates. Chem. Commun. 2012, 48, 449-451.

20. Liu, B.; Guo, Q.; Cheng, Y.; Lan, J.; You, J. Palladium-catalyzed desulfitative C-H arylation of heteroarenes with sodium sulfinates. Chem. Eur. J. 2011, 17, 13415-13419.

21. Zhao, F.; Tan, Q.; Xiao, F.; Zhang, S.; Deng, G. Palladium-catalyzed desulfitative cross-coupling reaction of sodium sulfinates with benzyl chlorides. Org. Lett. 2013, 15, 1520-1523.

22. Fleming, F.F.; Wang, Q. Unsaturated nitriles: Conjugate additions of carbon nucleophiles to a recalcitrant class of acceptors. Chem. Rev. 2003, 103, 2035-2078.

23. Kukushkin, V.Y.; Pombeiro, A.J.L. Additions to metal-activated organonitriles. Chem. Rev. 2002, 102, 1771-1802.

24. Rach, S.F.; Kuhn, F.E. Nitrile ligated transition metal complexes with weakly coordinating counteranions and their catalytic applications. Chem. Rev. 2009, 109, 2061-2080.

25. Larock, R.C.; Tian, Q.; Pletnv, A.A. Carbocycle synthesis via carbopalladation of nitriles. J. Am. Chem. Soc. 1999, 121, 3238-3239.

26. Ueura, K.; Satoh, T.; Miura, M. Rhodium-catalyzed arylation using arylboron compounds: Efficient coupling with aryl halides and unexpected multiple arylation of benzonitrile. Org. Lett. 2005, 7, 2229-2231.

27. Wong, Y.-C.; Parthasarathy, K.; Cheng, C.-H. Direct synthesis of arylketones by nickel-catalyzed addition of arylboronic acids to nitriles. Org. Lett. 2010, 12, 1736-1739. 
28. Tsui, G.C.; Glenadel, Q.; Lau, C.; Lautens, M. Rhodium(I)-catalyzed addition of arylboronic acids to (benzyl-/arylsulfonyl)acetonitriles: Efficient synthesis of $(Z)$ - $\beta$-sulfonylvinylamines and $\beta$-keto sulfones. Org. Lett. 2011, 13, 208-211.

29. Lindh, J.; Sjöberg, P.J.R.; Larhed, M. Synthesis of aryl ketones by palladium(II)-catalyzed decarboxylative addition of benzoic acids to nitriles. Angew. Chem. Int. Ed. Engl. 2010, 49, 7733-7737.

30. Hsieh, J.-C.; Chen, Y.-C.; Cheng, A.; Tseng, H.-C. Nickel-catalyzed intermolecular insertion of aryl iodides to nitriles: A novel method to synthesize arylketones. Org. Lett. 2012, 14, 1282-1285.

31. Wang, X.; Liu, M.; Xu, L.; Wang, Q.; Chen, J.; Ding, J.; Wu, H. Palladium-catalyzed addition of potassium aryltrifluoroborates to aliphatic nitriles: Synthesis of alkyl aryl ketones, diketone compounds, and 2-arylbenzo[b]furans. J. Org. Chem. 2013, 78, 5273-5281.

32. Wang, X.; Wang, X.; Liu, M.; Ding, J.; Chen, J.; Wu, H. Palladium-catalyzed reaction of arylboronic acids with aliphatic nitriles: Synthesis of alkyl aryl ketones and 2-aryl benzofurans. Synthesis 2013, 45, 2241-2244.

33. Zheng, H.; Zhang, Q.; Chen, J.; Liu, M.; Cheng, S.; Ding, J.; Wu, H.; Su, W. Copper(II) acetate-catalyzed addition of arylboronic acids to aromatic aldehydes. J. Org. Chem. 2009, 74, 943-945.

34. Qin, C.; Wu, H.; Chen, J.; Liu, M.; Cheng, J.; Su, W.; Ding, J. Palladium-catalyzed aromatic esterification of aldehydes with organoboronic acids and molecular oxygen. Org. Lett. 2008, 10, $1537-1540$.

35. Qin, C.; Wu, H.; Cheng, J.; Chen, X.; Liu, M.; Zhang, W.; Su, W.; Ding, J. The palladium-catalyzed addition of aryl- and heteroarylboronic acids to aldehydes. J. Org. Chem. 2007, 72, 4102-4107.

36. Zheng, H.; Ding, J.; Chen, J.; Liu, M.; Gao, W.; Wu, H. Copper-catalyzed arylation of arylboronic acids with aldehydes. Synlett 2011, 2011, 1626-1630.

37. Qin, C.; Chen, J.; Wu, H.; Cheng, J.; Zhang, Q.; Zuo, B.; Su, W.; Ding, J. One-pot synthesis of diaryl ketones from aldehydes via palladium-catalyzed reaction with aryl boronic acids. Tetrahedron Lett. 2008, 49, 1884-1888.

38. Lothrop, W.C.; Goodwin, P.A. New modification of the Ullmann synthesis of fluorene derivatives. J. Am. Chem. Soc. 1943, 65, 363-367.

39. Martinez-Viturro, C.M.; Dominguez, D. Synthesis of aza analogues of the anticancer agent batracylin. Tetrahedron Lett. 2007, 48, 4707-4710.

Sample Availability: Not available.

(C) 2014 by the authors; licensee MDPI, Basel, Switzerland. This article is an open access article distributed under the terms and conditions of the Creative Commons Attribution license (http://creativecommons.org/licenses/by/3.0/). 